\title{
A suggested intentional extraction of a wisdom tooth: Implies capacity for prosocial behaviour in Homo erectus
}

SADJ April 2020, Vol. 75 No. 3 p142 - p148

U Ripamonti ${ }^{1}$, JW Hoffman², C Ferretti ${ }^{3}$, LC Roden ${ }^{4}$

\begin{abstract}
Paleopathological assessment of a fossilized mandibular fragment (SK 45) of Homo erectus, unearthed at Swartkrans, South Arica, shows new bone formation within the alveolus of the third molar (M3), indicating that the individual lived after loss of that tooth.
\end{abstract}

As there is no evidence of mandibular maxillofacial damage compatible with acute traumatic expulsion of the tooth, it is possible that M3 was intentionally removed. Evidence of bone formation within the socket is supported by a density map of voxel values, representative of the atomic numbers of bone and breccia, constructed by microfocus scanning X-ray tomography $(\mu \mathrm{CT})$.

The newly formed material within the M3 alveolus has values less than the alveolar bone proper but which are significantly greater than breccia, indicating that the material is regenerating bone, and pointing to the possibly intentional extraction of M3.

How this was done and whether it was effected by the individual concerned or with the aid of community members, remains in the realms of speculation. The latter is more likely and may be an indication of the early origin

\section{Author affiliations:}

1. Ugo Ripamonti: MD, DDS, MDent, MFS, PhD (Med), Bone Research Unit, University of the Witwatersrand, Johannesburg, School of Clinical Medicine - Internal Medicine, Faculty of Health Sciences, 7 York Road, Parktown 2193, South Africa. ORCID Number: 0000-0002-6567-3594

2. Jakobus W Hoffman: BSc (Hons), South African Nuclear Energy Corporation (Necsa), South Africa.

3. Carlo Ferretti: BDS, MDent, FCD (SA) MFOS, Bone Research Unit, University of the Witwatersrand, Johannesburg, School of Clinical Medicine, Faculty of Health Sciences, Parktown, South Africa and Department of Maxillo-Facial and Oral Surgery, University of Pretoria, South Africa.

ORCID Number: 0000-0002-8862-391X

4. Laura C Roden: BSc, (Hons), PhD, Bone Research Unit, University of the Witwatersrand, Johannesburg, School of Clinical Medicine, Faculty of Health Sciences, Parktown, South Africa and School of Life Sciences, Faculty of Health Sciences, Coventry University, Coventry, CV1 2DS, UK

Corresponding author: Ugo Ripamonti

School of Clinical Medicine, Faculty of Health Sciences, 7 York Road,

Parktown 2193, South Africa.

Email: ugo.ripamonti@wits.ac.za

Author contributions:

1. Ugo Ripamonti: Conceptualised study, carried out data analyses, co-wrote manuscript - 50\%

2. Jakobus W Hoffman: Carried out Microfocus scanning X-ray tomography and data analyses, contributed to manuscript - $20 \%$

3. Carlo Ferretti: Contributed to manuscript - $10 \%$

4. LC Roden: Data analyses and co-wrote manuscript - $20 \%$ of prosocial behaviour at the Plio-Pleistocene boundary, resulting in a concerted action of the Homo clade to assist an individual in severe distress.

\section{Keywords}

Homo erectus, Swartkrans, SK 45 mandibular remains, wisdom tooth, intentional extraction, alveolar bone regeneration in hominine evolution, prosocial behaviour.

\section{INTRODUCTION}

Clearly, medical and surgical procedures at the Early Pleistocene boundary are unknown and the subject of speculative reasoning only.

One of the behavioural traits of Homo sapiens that separates us from our closest primate relatives, and, some paleontologists believe, from early hominins, is that of compassion and pro-social emotions that promote behaviour which will benefit another. ${ }^{1}$

Fossil specimens indicating survival of individuals with severe ante-mortem traumas ${ }^{2}$ and tooth loss $^{3,4}$ have been interpreted as evidence of compassion and conspecific care in early hominins. However, comparative analyses advise caution in these interpretations as similar disabilities have been observed in non-human primates who have survived without nonspecific care.5,6

Unlike the virtually edentulous Dmanisi specimen which displayed extensive ante-mortem tooth loss and extensive alveolar remodelling and atrophy (specimens D3444 and D3900), ${ }^{4}$ SK 45 retained M1 and M2, surviving the loss of M3. The survival of the Dmanisi individual for many years after the loss of most of its teeth was attributed to altered diet or care by other individuals. ${ }^{4}$

The concept of 'behavioural modernity' or 'human uniqueness' is an issue which drives the study of human evolution beyond the basic comparison of fossil morphology to seeking an understanding of our origins.

The social behaviour of early Homo species is problematic to measure or envisage as direct evidence is not preserved. Foley and $\mathrm{Gamble}^{7}$ considered paleontological and archaeological evidence in the context of changing climate and selective pressures to make inferences about human-specific behavioural changes that may have lead to the evolution of 'human society'. 
At the Early Plio-Pleistocene boundary (2-1.5 million years Myr B.P.) the inclusion of meat in early Homo diet would have provided for a lower seasonal variation in food availability.

Significantly in societal evolutionary terms this relative security promoted a delayed life-history strategy, allowing greater bonding between males and females, ${ }^{7}$ and perhaps also between members of the social group. This period was also characterized by the use of tools for hunting, gathering and food preparation, evidence of this gathered from multiple sites including Swartkrans cave, South Africa. ${ }^{7-11}$

Was it at this transition in the social history of Homo that a 'theory of mind' was developed, allowing social engagement with orders of intentionality? ${ }^{12}$ Our purpose here is to provide a detailed description of the analytical examination of SK 45, supporting the possible intentional extraction of M3 and thus offering paleopathological and paleobiological evidence for an early origin of prosocial behaviour to relieve pain in another amongst Homo erectus at Swartkrans, South Africa.

\section{MATERIALS AND METHODS}

Mandibular fossil SK 45, confined on evolutionary and faunal grounds to 1.5 - 1.2 million years before the present (Myr B.P.), ${ }^{13,14}$ was unearthed at Swartkrans cave, Krugersdorp, South Africa, and described in detail by Broom and Robinson ${ }^{15}$ and Robinson. ${ }^{16}$

Originally designated as belonging to species Telanthro-

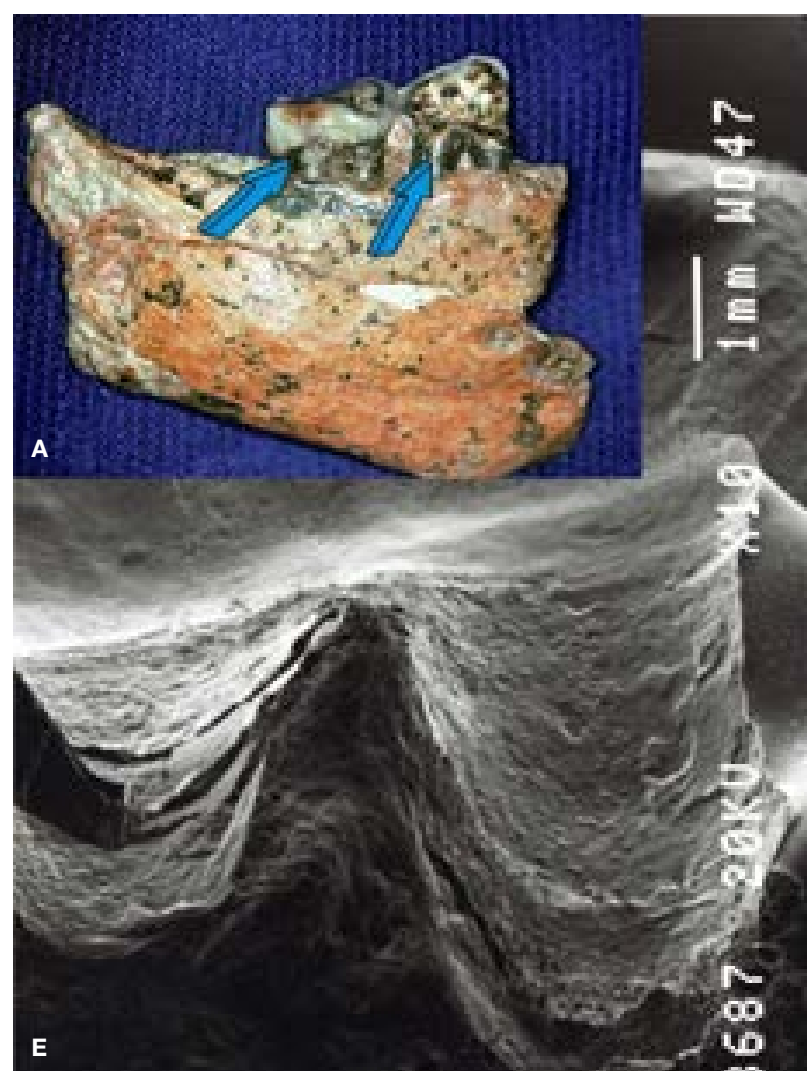

Figure 1. Buccal (A), and lingual (B, C and D) views of SK 45 mandibular fragment of Homo erectus highlighting significant vertical bone loss affecting M1 and M2 (light blue arrows) with Class II furcation defects of the affected molars. Arrows in $\mathrm{C}$ and $\mathrm{D}$ indicate the remaining alveolar pus capensis, the specimen was later attributed to Homo erectus. ${ }^{17,18}$ SK 45 is from an adult individual of Homo erectus and consists of the right mandibular fragment with molars 1 (M1) and M2 (Figs. 1A, 1B). SK 45 is housed at the Ditsong National Museum of Natural History, Pretoria.

\section{Microfocus scanning X-ray tomography}

Scans of SK 45 were initiated with 1000 X-ray projections in $360^{\circ}$ using a Nikon XT H 225 System which is housed at the MIXRAD facility at the South African Nuclear Energy Corporation (NECSA). ${ }^{19}$ Full-view scans of the whole sample as well as scans focused on the region of interest (the alveolus of $\mathrm{M} 3$ distal to M2) were performed at $140 \mathrm{kV}$ and $70 \mu \mathrm{A}$ which allowed for a spatial resolution of $34 \mu \mathrm{m}$. The reconstruction process transformed the 2D projection images into a 3D rendering which was analyzed in volume rendering software (VGStudio MAX, Volume Graphics $\mathrm{GmbH}$, Heidelberg, Germany).

A density map of voxel values representative of the atomic numbers of different materials within the specimen was constructed using $\mu \mathrm{CT}$. Several rectangular areas were sampled independently on different dates and examined in reformatted sagittal images to determine a densitometry map across the newly formed material in the alveolus of M3 (Fig. 2C), the mandibular bone adjacent to the alveolus (Fig 2), as well as breccia evidenced coronal to the residual bony housing of $\mathrm{M} 1$ and $\mathrm{M} 2$ (Figs. 1B, C, D) and through the furcations of $\mathrm{M} 1$ and $\mathrm{M} 2$.

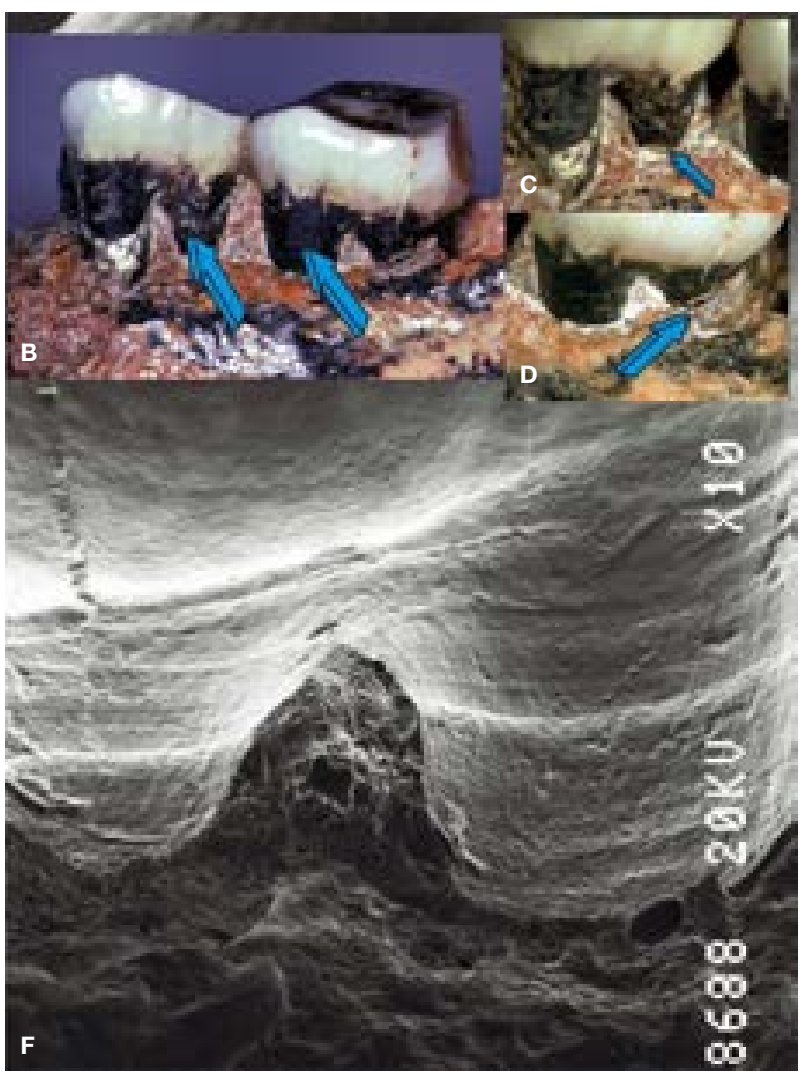

bony housing. (E, F): Scanning Electron Macrographs (SEM) of the buccal aspect of M1 and M2 showing the severe alveolar bone loss and Class II furcation exposure of the affected molars. 


\section{Scanning Electron Microscopy}

After cleaning $\mathrm{M} 1$ and $\mathrm{M} 2$ with acetone, vinyl polysiloxane impression material (3M Express ${ }^{\circledR}$, Regular Set, type 1 very low viscosity) was applied to the buccal surfaces of SK 45.

A mixed base and catalyst putty of vinyl polysiloxane (type 1 very high viscosity), previously set in a tray contoured for SK 45, was adapted over the low viscosity material to allow optimal surface distribution and penetration, particularly within the exposed furcations of SK 45.

The negative replica was mounted on a SEM aluminum tub, coated with gold palladium and examined using a JEOL scanning electron microscope. ${ }^{20}$ The replicas provided positive and reliable images (Figs. 1E and $\mathrm{F}$ ) with no indication of distortion up to 250X.20

\section{RESULTS AND DISCUSSION}

The specimen from an adult Homo erectus individual consists of the right mandibular fragment with $\mathrm{M} 1$ and M2 in situ (Figs. 1A, B) and showing severe attrition of the occlusal surfaces. It will be observed that SK 45 suffered from periodontitis as evidenced by the severity of alveolar bone loss with buccal and lingual furcation exposure (Fig. 1).

Loss of alveolar bone, as measured with a periodontal probe from the cemento-enamel junction to the remaining bony housings extended from 4 to $8 \mathrm{~mm}$ with severe bone loss lingually (Figs. 1A, B, C, D).

The extent of bone loss and furcation exposure, now filled with calcite and breccia (Figs. 1A, B, C, D) is pronounced when measured from the cementoenamel junctions of both mandibular molars (Figs. 1A, B, C, D).

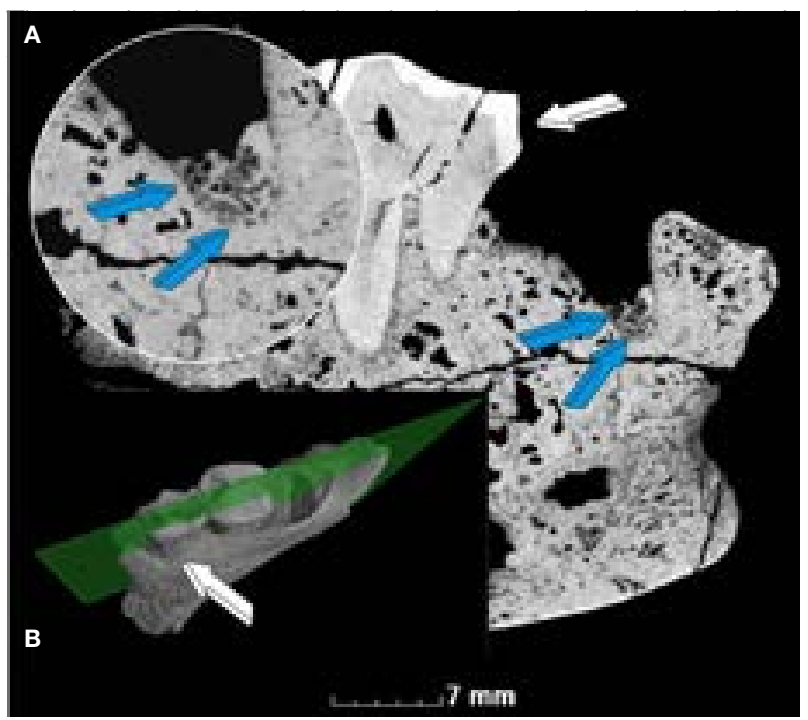

Figure 2. Sagittal $\mu C T$ reformatted images of SK 45 across the molar area as shown in the 3D virtual image $A$, analyzing the alveolar bony housings of M2, M3 and the alveolus of the missing M3. White arrow in A indicates the enamel contact point of M2 with the over-extruded and tilted M3. White arrow in $B$ points to severe lingual bone loss affecting the M1 mesial root. Blue arrows in $\mathrm{B}$ and $\mathrm{C}$ indicate the profile and the well preserved tissue boundaries of the alveolus of M3 ( $C$ inset) containing mineralized material interpreted as newly generated bone after M3 avulsion.
Exposure was confirmed by non-invasive micro focus X-ray computed tomography $(\mu \mathrm{CT})$ analyses (Fig. $3 \mathrm{C}) .^{19}$ The digitized $\mu \mathrm{CT}$ scan images (Fig. 2) reveal the identifiable outline of the $\mathrm{M} 3$ alveolus filled with incompletely remodelled but mineralized bone (Fig. 2B, C). The 2D projection images were transformed into a $3 \mathrm{D}$ rendering (Fig. 3A) which shows the alveolus of M3 (Fig. 3B).

According to Robinson, ${ }^{16} \mathrm{M} 3$ could not have exceeded $10.5 \mathrm{~mm}$ in length. $\mu \mathrm{CT}$ analyses show that the root was conoidal and distally located (Fig. 2B). The tomographic image also shows the occlusal contact point of the previously housed $\mathrm{M} 3$ with the distal enamel surface of M2 (Figs. 2B white arrow; 3B light blue arrow).

The retro-molar area shows the regenerating alveolar bone within the alveolus of the missing M3 (Figs. 2B, C light blue arrows). Wisdom teeth are often extracted in extant Homo sapiens due to painful infections, particularly when the tooth is partially erupted.

In Homo, impaction and misalignment of $\mathrm{M} 3$ has also been ascribed to the reduction of the mandibular body during evolution and speciation of the Homo clade at the Early Pleistocene boundary. ${ }^{16,21}$ The implication of possible third molar impactions in the hominid fossil record has also been discussed. ${ }^{22}$

Differential diagnosis to determine the cause of M3 loss must include avulsion following severe trauma. However, in the absence of a mandibular fracture an avulsion of a third molar is not feasible and as no evidence of mandibular fracture is seen in SK 45 we may safely discount traumatic avulsion as a cause.

Neither gross nor $\mu \mathrm{CT}$ analyses support traumatic loss, particularly because of the substantial remaining alveolar bone outlining the remaining alveolus (Figs. 2B, C).

A further possibility is spontaneous exfoliation following a protracted course of periodontal disease. This scenario is also less likely as this is a protracted event, with ossification and mineralization occurring as the tooth slowly

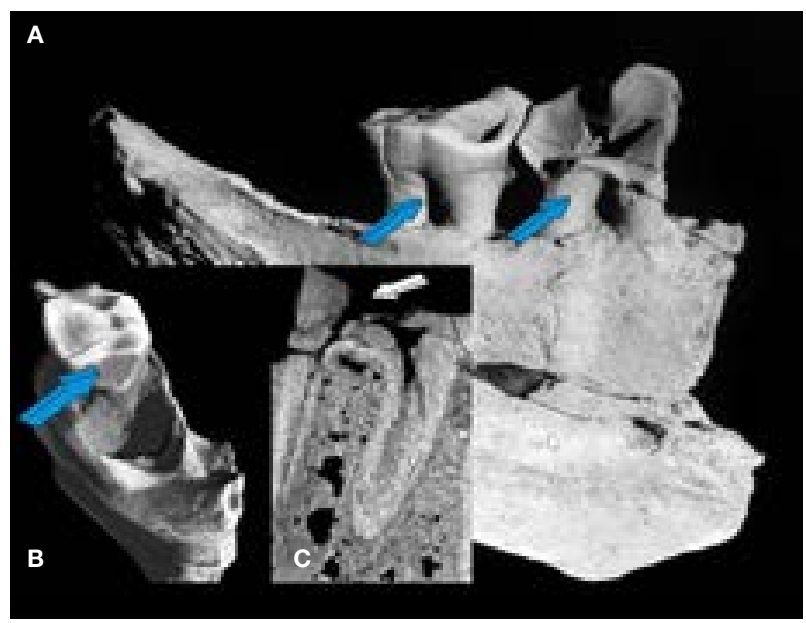

Figure 3. (A, B) virtual volume of SK 45 Homo erectus. Light blue arrows in (A) point to the evidence of periodontal bone loss with substantial furcation exposure; $(B)$, occlusal distal view of the alveolus of the missing M3 with the enamel contact point of M2 (arrow). (C), sagittal $\mu$ CT image of M1: (white arrow) indicates severe wear of the enamel with pulp exposure of the mesial root. 
exfoliates, eliminating the radiographic picture of a clearly identifiable socket. The radiographic remnant would be a completely healed alveolus without vestiges of the socket.

In addition, the rather large triangular space between the distal root of $\mathrm{M} 2$ and the alveolus was likely to have been covered by gingivae which probably also covered most of M3. The presence of such alveolar anatomical structures makes the expulsion or the acute exfoliation of the tooth very unlikely.

Importantly, the presence of a well-defined alveolus as seen by $\mu \mathrm{CT}$ scans with a distinct corticalized bone profile on M3 makes it very unlikely that the periodontal pathology affecting M1 and M2 (Figs. 1A, 1B) would have caused acute periodontal episodes to induce the spontaneous exfoliation of M3. Another possibility is the post-mortem loss of $\mathrm{M} 3$ following precipitation of the mandible in the cave deposits of Swartkrans or later during fossilization.

To further investigate the material present within the M3 alveolus, the density map of voxel values representative of atomic number of different materials, constructed using $\mu \mathrm{CT}$ revealed voxel values $( \pm$ standard error) of the mandibular alveolar bone proper (30250 \pm $461)$, breccia $(27018 \pm 469)$ and the $\mathrm{M} 3$ alveolar region of interest (28792 \pm 930 ) (Figs. 2B, 2C). These are all statistically significantly different from each other $(\mathrm{p}<$ 0.005) as was shown in a two tailed Welch's t-test. While the voxel values of the material within the alveolus of M3 are less dense than the alveolar bone $(p=0.047)$, the material is however denser than the breccia $(p=0.0006)$. The differences in density and thus the degree of mineralization of the examined bone and breccia area suggests that the material within the alveolus of M3 may be regenerating alveolar bone.
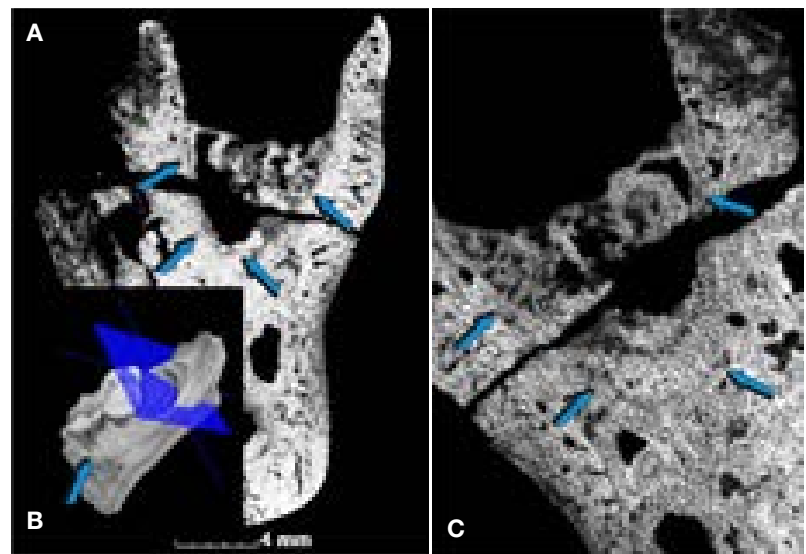

Figure 4. Coronal tomographic reformatted images of SK 45 through the alveolus of M3.

A). 3D tomogram of SK 45 indicating the location of the coronal tomographic reformatted images and the remaining lingual alveolar bony housing: M1 is affected by severe furcation exposure with pronounced mesio-lingual alveolar bone loss (arrow) of the mesial root of M1.

B). Reformatted image as representative of the coronal plane shown blue in $\mathrm{A}$.

C). Coronal reformatted image more distally located and slightly more disto-mesially oriented across the alveolus. Arrows in B and C show the preserved and maintained boundaries of the alveolus with the surrounding mandibular bone, indicating that the mineralized material within the alveolus is not breccia but regenerated alveolar bone after the avulsion of M3; this is also supported by the difference in densitometric grey values as compared to breccia material (see text).
Density maps of voxel values were repeated at different times, and the voxel values of the mandibular alveolar bone proper, breccia and of the region of interest were: $18780 \pm 207.14,16309 \pm 296.16$ and $17373 \pm 72.78$ confirming the previous data taken at different regions of interest across different tomograms of the specimen.

The data recorded at different sites from multiple measurements at different time-points show that the region of interest within the alveolus of the missing $M 3$ is statistically different from breccia. The voxel values of the region of interest are also significantly different from the measures of alveolar bone and show smaller values. This indicates that the region of interest is partially filled with mineralizing newly formed bone.

Statistically significant differences do exist between the fossilized regions which were examined, including the region of interest. However, voxel values are often considered unreliable when differences are not truly major, even though statistically significant, particularly when highly fossilized specimens are evaluated. SK 45 fits that context. It may be pertinent that voxel values were also different when measured at different time points.

The first measurements as reported above of voxel grey values are merely representations of the density on a 16-bit grey scale; values were obtained by simply opening the data in the software package VGStudio Max 3.2. Subsequent measurements were designed to maximize the effect of different voxel grey values. The sample data was re-imported into the software with a calibration procedure where the background grey values and highest density material grey values were explicitly specified.

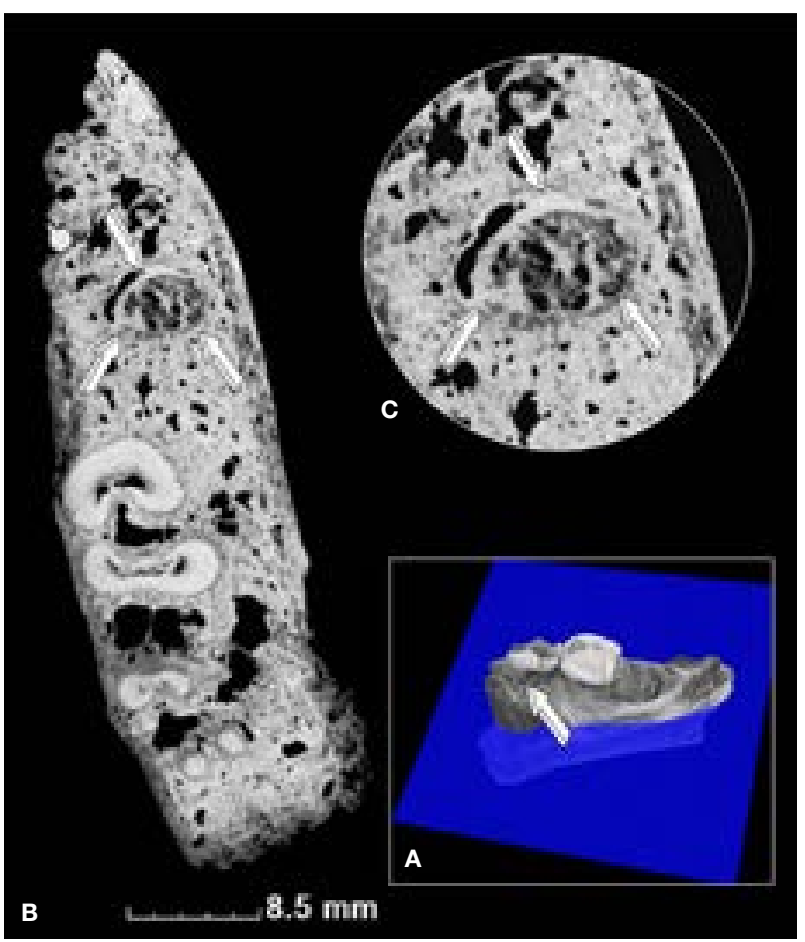

Figure 5. Transverse $\mu$ CT slice images of SK 45. A, 3D virtual image indicating the location of the transverse tomographic slice as shown in $\mathrm{B}$ depicting the severe furcation exposure with alveolar bone loss (arrow) of M1. B, C, Transverse $\mu$ CT slice images of the alveolus of M3 showing the maintained and well-preserved boundaries (arrows in B and C) of the mineralized material, interpreted as bone, that had formed within the alveolus with surrounding mandibular bone issue. 
These values were specified by selecting a region of interest in a view of the sample and were consequently chosen in air pores within the sample with the enamel visible in the same view. Both operations allowed for the same conclusions regarding the densities of the various regions, which were highly reproducible between sets of measurements.

Progressive mesial to distal coronal tomographic slices of the SK 45 mandible distal to M2 shows the presence of preserved anatomical boundaries within the alveolus (Figs. 4B, C). Sagittal (Fig. 2), coronal (Fig. 4) and transverse (Fig. 5) $\mu \mathrm{CT}$ scan images of the region of interest distally to $M 2$ show in more detail the alveolus of the missing M3 with dense mineralized material within the socket and with continuous preservation of the anatomical boundaries of the alveolus (arrows in Figs. 2, 4, 5).

The tomographic images also present a trabecular-like pattern in the region of interest of the M3 alveolus, noticeably different when compared with the appearance of the breccia deposits.

On axial tomograms (Fig. 5) the newly deposited material presents a well-defined corticalized area of the newly formed bone with trabeculations across the alveolus compatible with (or suggesting) deposition and remodelling (Figs. 5B, C).

For comparative purposes, the other Swartkrans Homo erectus mandible, SK 15, was also examined and scanned by $\mu C T$. Several teeth are missing in SK 15 anteriorly on both hemi-mandibles. The $\mu \mathrm{CT}$ showed the empty alveolus of the left first premolar (P1) with breccia

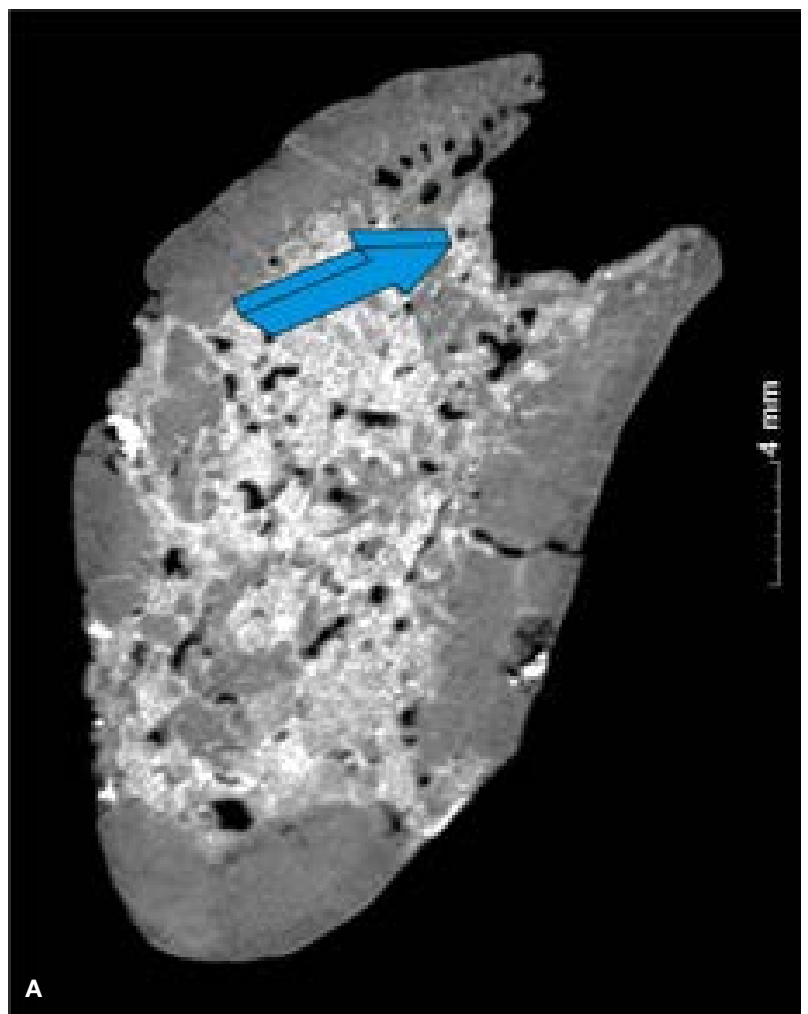

Figure 6. Coronal $\mu \mathrm{CT}$ slices of Homo erectus SK 15 mesial to the left $\mathrm{M} 1$ showing the alveolus of the missing $2^{\text {nd }}$ bicuspid (arrow A) with some breccia outlining the profile of the alveolus (arrow). forming within the alveolus (Fig. 6) and merging with the invading breccia that had formed within the intercortical trabecular area of the mandible (Fig. 6A).

Further mesially, coronal tomographic slices showed the total blending of breccia within the intercortical area with no outlines whatsoever of the empty alveoli after postmortem loss of the premolars (Fig. 6B).

Finally, differential diagnosis must also include the intentional extraction of $\mathrm{M} 3$. The tomographic images of SK 45 (Figs. 2B, 3, and 4) confirm that the use of a straight tool inserted between M2 and M3 with appropriate rotational and lever movements would have allowed extraction of the offending third molar.

Indeed, Fig. 2A shows a depression distal to M2 with indentation of the alveolar bone possibly reflecting the tight insertion with rotational movement of an osteodontokeratic tool being used for the extraction of M3.

In-depth studies on hand morphology, tool-making, toolusing and precision grips of early hominids and Homo species at the Early Pleistocene boundary at Swartkrans have suggested not only the use of tools but also that the tools had precision grips. ${ }^{23-25}$ The most likely diagnosis is, therefore, the intentional extraction of $\mathrm{M} 3$.

If this diagnosis is correct, why in the life of SK 45 did this molar, which was functional for a long time based on the facet contact point with M2 (Fig. 2B), suddenly became a problematic tooth needing extraction? In extant Homo sapiens, long quiescent third molars may cause symptoms either due to acute pulpitis secondary

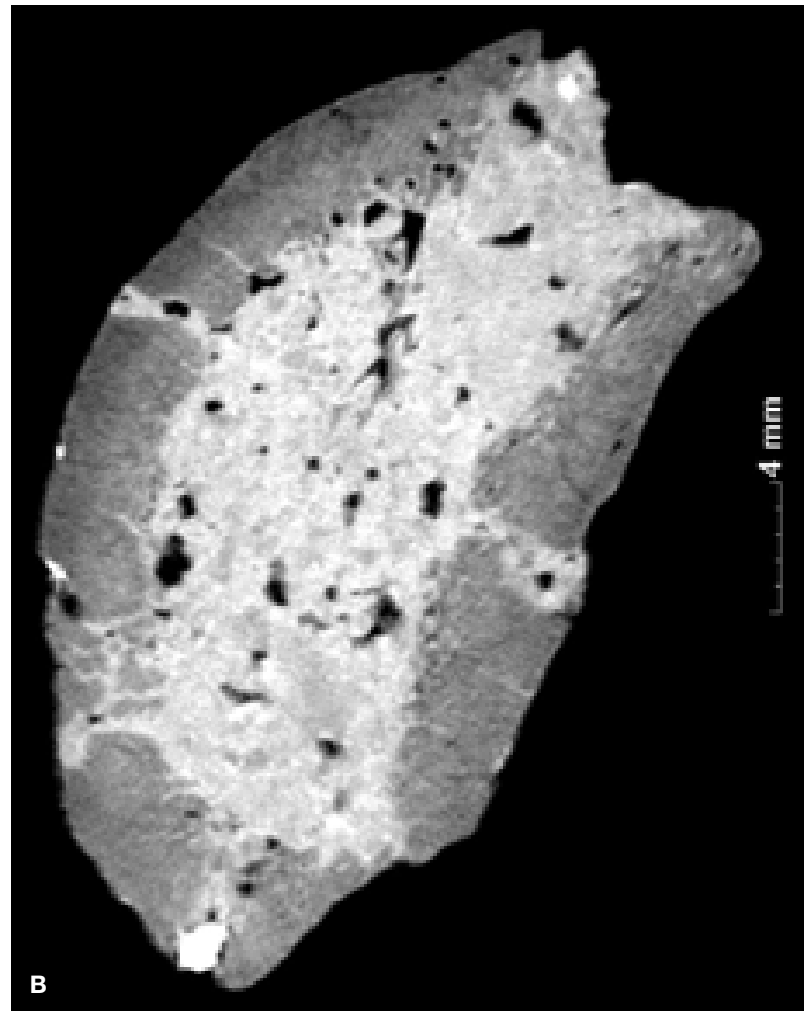

(B) further anterior $\mu \mathrm{CT}$ slice to M1 across the edentulous anterior mandible shows breccia penetrating the alveoli and the corticocancellous medullary spaces of the body of the mandible, with no evidence of alveolus boundaries. 
to decay of a partially erupted third molar or the development of acute pericoronitis. It is thus conceivable that a partially erupted third molar in SK 45 may have become symptomatic.

The angle of the residual socket and the interdental wear facet confirm that M3 was not parallel to M2 (which is the most common relationship in a fully erupted tooth) but rather lying mesioangular and thus likely to have been partially erupted.

The root morphology (single rooted and conical) would also make this tooth less challenging to remove. The paleopathologic evidence of the lost M3 in SK 45, correlated with experience in extant patients, favours the diagnosis of intentional extraction. The hominid SK 45 survived the surgical extraction of $\mathrm{M} 3$ as shown by the presence of regenerating bone within the alveolus (Figs. 2B, C).

The hypothesized surgical intervention by the individual or with the assistance of group members would indicate a refined group support and care, suggesting a social community network capable of prosocial acts. This would reflect not only a lack of discrimination but also a strong social cohesion amongst this early Homo species.

The suggestion of a lack of discrimination with possible social integration has been made after the diagnosis of a case of pre-pubertal periodontitis in a juvenile Australopithecus africanus specimen. ${ }^{26}$

Tantalizingly, this scenario raises an even more interesting probability: the capacity of the hominids to correlate physical symptoms with anatomical structures, to develop a therapeutic intervention for the resolution of those symptoms and, at the same time, to use a selected tool with precision and power grasping capabilities.

This implies a significantly higher intellectual development than has been considered likely heretofore. The mirror neuron system (MNS), first discovered in non-human primates, ${ }^{27,28}$ is activated in an animal in response to the observation of hand or facial gestures in another. The MNS is thought to function in empathy ${ }^{29,30}$ which is essential in the development of an altruistic society. ${ }^{31}$ Furthermore, the MNS facilitates complex communication through facial expressions and referential vocalizations, in the absence of spoken language. ${ }^{28,29}$

\section{CONCLUSIONS}

The presented data indicate new bone formation in the alveolus of a third molar of Homo erectus, suggesting an apparent intentional extraction of a wisdom tooth.

This proposal is made on anatomical-pathologic findings as evidenced by $\mu \mathrm{CT}$ ultimately suggesting that Homo erectus had not only social structures to assist individuals in discomfort, but also highly evolved problemsolving capacities which extended beyond the development and use of tools for hunting and food preparation.

This supports the idea that the capacity for behavioural variability $^{32}$ is a characteristic of more than one hominin species and evolved prior to the origin of Homo sapiens. The presented $\mu C T$ scan images are the first to show induced bone in hominine evolution and speciation at the emergence of the Homo clade.

\section{Acknowledgements}

We thank Bona Nyawose and Stephany Potze then of the Ditsong National Museum of Natural History, Pretoria, for cooperation in the examination of SK 45 and SK 15 specimens; F.C. de Beer of Necsa, South Africa, for help with the microfocus X-ray computed tomography; Jean-Claude Petit and Ansuhya Magan for constructive criticism.

The South African National Research Foundation Research Infrastructure Support Programme (NRF-RISP) funded the purchase of the MIXRAD Nikon XT H 225 system: \#72310. This work is supported by the University of the Witwatersrand, Johannesburg and by ad hoc grants by the Bone Research Laboratory, School of Clinical Medicine - Internal Medicine, Faculty of Health Sciences.

\section{References}

1. Hill K, Barton M, Hurtado M. The emergence of human uniqueness: characters underlying behavioural modernity. Evol Anthropol. 2009; 18: 187-200.

2. Trinkaus E, Zimmerman MR. Trauma among the Shanidar Neandertals. Am J Phys Anthropol. 1982; 57: 61-76.

3. Lebel S, Trinkaus E, Faure M, Fernandez P, Guérin C, Richter D, Mercier N, Valladas H, Wagner GA. Comparative morphology and paleobiology of Middle Pleistocene human remains from the Bau de l'Aubesier, Vaucluse, France. Proc Natl Acad Sci. U.S.A. 2001; 98, 11097-102.

4. Lordkipanidze D, Vekua A, Ferring R, Rightmire GP, Agusti J, Kiladze G, Mouskhelishvili A, Nioradze M, Ponce de León MS, Tappen M, Zollikofer CP. Anthropology: the earliest toothless hominin skull. Nature. 2005; 434: 717-8.

5. DeGusta D. Comparative skeletal pathology and the case for conspecific care in Middle Pleistocene Hominids. J Archaeol Sci. 2002; 29: 1435 -8.

6. Hublin JJ. The prehistory of compassion. Proc Natl Acad Sci. U.S.A. 2009; 106, 6429-30.

7. Foley R, Gamble C. The ecology of social transitions in human evolution. Phil Trans R Soc. B. 2009; 364: 3267-79.

8. Blackwell LR, d'Errico F. Evidence of termite foraging by Swartkrans early hominids. Proc Natl Acad Sci. U.S.A. 2001; 98: 1358-63.

9. Brain CK. (Ed.) 1993. Swartkrans: A Cave's Chronicle of Early Man. Transvaal Museum Monograph No. 8.

10. Dart RA. Australopithecus prometheus and Telanthropus capensis. Am J Phys Anthropol. 1955; 1: 67-96.

11. Wolberg DL. The hypothesized osteodontokeratic culture of the Australopithecinae: A look at the evidence and the opinions. Curr Anthropol. 1970; 11: 23-37.

12. Premack D, Woodruff G. Does the chimpanzee have a theory of mind? Behav Brain Sci. 1978; 1: 515-26.

13. Vrba ES. Chronological and ecological implications of the fossil Bovidae at the Sterkfontein Australopithecine site. Nature. 1974; 250: 19-23.

14. Vrba ES. Some evidence of chronology and paleoecology of Sterkfontein, Swartkrans and Kromdraai from the fossil Bovidae. Nature. 1975; 254: 301-4.

15. Broom R, Robinson JT. Man contemporaneous with the Swartkrans ape-man. Am J Phys Anthropol. 1950; 8: 151-7.

16. Robinson JT. Telanthropus and its phylogenetic significance. Am J Phys Anthropol. 1953; 11: 445-87. 
17. Robinson JT. The Australopithecines and their bearing on the origin of man and of stone tool-making. S Afr J Sci. 1961; 57: 3-16.

18. Robinson JT. The origin and the adaptive radiation of the Australopithecines. In Kurth G. (Ed.), Evolution und Hominisation. Fischer Verlag, Stuttgart. 1961; 120 - 40.

19. Hoffman JW, De Beer FC. Characteristics of the MicroFocus X-ray Tomography Facility (MIXRAD) at Necsa in South Africa. 18 ${ }^{\text {th }}$ World Conference on Non-destructive Testing http://www.ndt.net/articlewcndt2012/papers/37_wcndtfinal00037.pdf

20. Ripamonti U, Petit J-C, Grossman ES. The hard periodontal tissues of the Australopithecinae. Proceedings of the Electron Microscopy Society of Southern Africa.1989; 19: 141-2.

21. Calcagno JM, Gibson KF. In: Kelley, M.A., Larsen, C.S. (Eds.), Advances in Dental Anthropology. Alan R. Liss, New York, 1991; 59-76.

22. Gibson KR, Calcagno JM. Possible third molar impactions in the hominid fossil record. Am J Phys Anthropol. 1993; 4, 517-21.

23. Marzke MW, Shacley SM. Hominid hand use in the Pliocene and Pleistocene: evidence from experimental archaeology and comparative morphology. J Hum Evol. 1986; 15: 439-60.
24. Marzke MW. Precision grips, hand morphology, and tools. Am J Phys Anthropol. 1997; 102: 91-110.

25. Susmann RL. Hand function and tool behaviour. J Hum Evol. 1998; 35: $23-46$.

26. Ripamonti U. Paleopathology in Australopithecus africanus: a suggested case of a three million-year-old prepubertal periodontitis. Am J Phys Anthropol. 1988; 2; 197-210.

27. Gallese V, Fadiga L, Fogassi L, Rizzolatti G. 1996. Action recognition in the premotor cortex. Brain 1996; 119: 593-609.

28. Rizzolatti G, Fadiga L, Gallese V, Fogassi L. Premotor cortex and the recognition of motor actions. Brain Res. 1996; 3 : 131-41.

29. Parr LA, Waller BM, Fugate J. Emotional communication in primates: implications for neurobiology. Curr Opin Neurobiol. 2005; 15: 716 - 20.

30. Rizzolatti $G$. The mirror neuron system and its function in humans. Anat Embryol (Berl). 2005; 210: 419-21.

31. de Waal FBM. Putting the altruism back into altruism: The evolution of empathy. Annu Rev Psychol. 2008; 59: 279-300.

32. Shea JJ. Homo sapiens is as Homo sapiens was. Curr Anthropol. 2011; 52: 1-35.

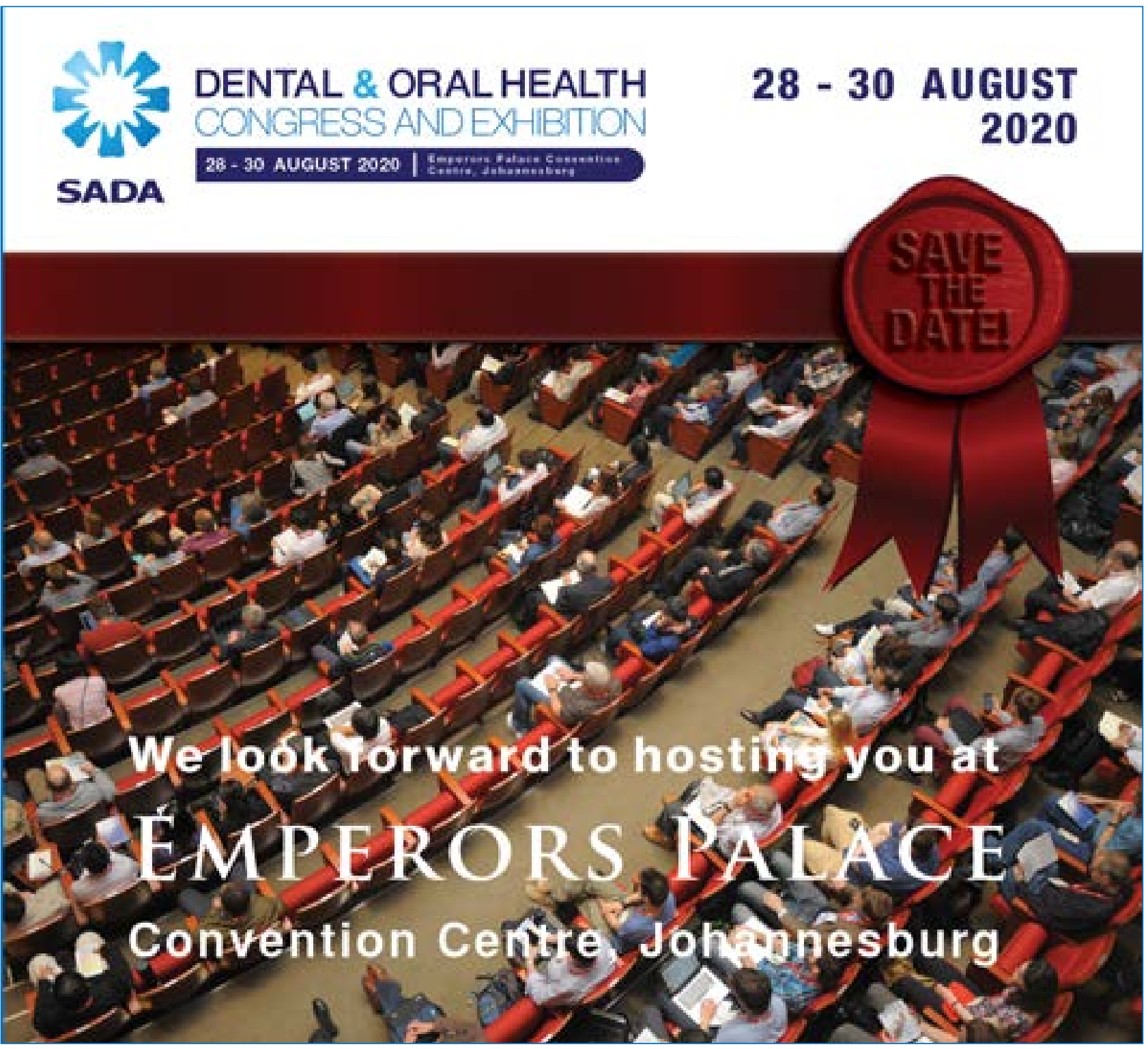

